\title{
Postburn Microstomia Prevention Using an Appliance Providing Simultaneous Horizontal and Vertical Adjustable Forces
}

\author{
Gregory S. Antonarakis, DDS, MSc, PhD, ${ }^{*} \uparrow$ Sandra Fastlicht, DDS, MSc, $\uparrow$ \\ Taras Masnyi, DDS, $\uparrow$ and Bryan Tompson, DDS, DPaedo, DOrth, FRCD $(C) \dagger$
}

\begin{abstract}
Microstomia, an abnormally small oral orifice, is a complication of perioral facial burns. In this case, contraction of the circumoral tissues and hypotonia of the musculature is responsible for this microstomia, which can produce aesthetic and functional impairment with eating, swallowing, communication (speech and facial expressions), compromised dental care and maintenance due to limited oral access, social interactions, and psychological well-being. Conservative management involves providing physical resistance to scar contracture, with opposing horizontal and vertical circumoral forces by means of appliances that aim to stretch the commissures and fibrotic muscles. Numerous appliances, either intraoral or extraoral, have been described to prevent or treat microstomia by delivering a static or dynamic stretch horizontally or vertically, with most designed to stretch the mouth horizontally. Finding a comfortable effective way to stretch the mouth vertically has proved to be a challenge. This article describes the fabrication of a dynamic commissural appliance, constructed using acrylic resin and expansion screws, which provide simultaneous horizontal and vertical circumoral forces. This appliance is constructed easily and inexpensively without the need for taking impressions, can be adjusted so that it is almost painlessly inserted, and is progressively activated. It is convenient for use because the patient controls the pressure that is applied by the appliance. Its use in a case is described where the appliance has improved mouth opening and consequently functional outcomes. (J Burn Care Res 2017;38:e977-e982)
\end{abstract}

After a thermal, electrical, or chemical burn to the face involving the oral cavity or its surroundings, prevention of postburn microstomia is of utmost importance. If proper treatment is not instituted immediately, such perioral facial burns, either partial or full thickness, can result in tissue scarring and contraction. ${ }^{1}$ This alteration to the perioral soft tissues can lead to hypertrophic scarring around the lips and contracture of the commissures, as well as a decrease in sphincteral action of the orbicularis oris muscle and hypotonia of the musculature, deforming

\footnotetext{
* Department of Orofacial Rebabilitation, University of Geneva School of Dental Medicine, Switzerland; and TDepartment of Dentistry, Hospital for Sick Children, Toronto, Ontario, Canada.

Address correspondence to Gregory S. Antonarakis, DDS, MSc, PhD, Department of Orofacial Rehabilitation, University of Geneva School of Dental Medicine, 19 rue Barthélemy-Menn, 1205

Geneva, Switzerland.E-mail:gregory.antonarakis@unige.ch. Copyright (C) 2017 by the American Burn Association 1559-047X/2017
}

DOI: $10.1097 / B C R .0000000000000523$ the mouth commissures leading to microstomia. ${ }^{2}$ Microstomia is characterized by a decrease in the size of the oral aperture that is not defined by specific size criteria. Diagnosis is often determined by its effect on function and appearance.

Adequate oral aperture and a correct range of motion are essential for mastication, feeding and nutrition, swallowing, speech and communication, proper oral hygiene, facial expressions, and social relationships, which are all affected by microstomia. ${ }^{3,4}$ Aesthetics, psychological well-being, and quality of life are compromised as a result. Moreover, difficulty in oral hygiene results in higher incidence of oral diseases such as caries, periodontal diseases, other oral infections, and halitosis, which are difficult to treat due to the limited access. ${ }^{5,6}$ Microstomia can also inhibit proper dental and skeletal development in a growing individual. ${ }^{7}$

Preventing postburn microstomia helps prevent the detrimental effects on these important daily oral functions. By applying opposing horizontal and vertical 
circumoral forces to scar contracture, one can try and manage the scarring process. A variety of interventions, intended to reduce scarring and maintain function have been proposed, including positioning, pressure, various appliances, and oral physiotherapy exercises. ${ }^{8,9}$ If this early conservative treatment is not provided, the resultant contracture may require later surgical reconstruction, such as surgical release of the oral commissures (commissuroplasty), which is invasive, complex, and not without side effects. ${ }^{10}$ After surgical correction, the tissue is replaced by fibrous connective collagen tissue, which is subject to constant contraction for months, and together with the action of the orbicularis muscle may result in the recurrence of microstomia. ${ }^{11} \mathrm{~A}$ conservative appliance-therapy approach is thus preferred with no surgical consideration for approximately 1 year since it may take this long for the postburn scar to become stable. ${ }^{12}$

Appliance-based procedures for treating microstomia use either static or dynamic appliances to provide resistance to scar contraction and promote stretching and mobility of the circumoral tissues and fibrotic muscles. ${ }^{13} \mathrm{~A}$ wide range of protocols exist with regard to appliance design, initiation of the appliance, frequency, and duration of use, ${ }^{4}$ but all are designed to reduce scarring and keep normal functions. Static appliances have no movable parts once in place. ${ }^{4}$ Dynamic appliances exert pressure that is adjustable permitting progressive controlled tissue stretching and are either intraoral or extraoral. ${ }^{13}$

Selection of an appropriate microstomia prevention appliance for a patient should be based on factors such as age, type and extent of injury, the condition of the dentition, the patient's ability to comply, comfort, durability, safety, expense, ease of design and fabrication, and need for repair and readjustment. ${ }^{12}$ Compliance is perhaps the most important factor for determining the appropriateness of an appliance best suited for the individual patient given that professionals find that poor compliance is the number one problem in treating patients with perioral facial burns. ${ }^{13}$ For these reasons, appliances must be well tolerated by the patient in order to avoid compliance problems related to appliance discomfort.

Microstomia prevention appliances used with patients having perioral burns need to stretch vertically, horizontally, and obliquely to provide effective contraction management in more than one plane. ${ }^{14,15}$ The majority of appliances used however address only the horizontal component of mouth opening and much less so the vertical contracture that occurs. ${ }^{4,15}$

Although there is debate regarding the best course of treatment for facial burns, there is agreement that early appliance use (within approximately 2 weeks of initial injury) before substantial healing has begun decreases the need for surgical reconstruction, and appliance wear should continue until the scar is mature. ${ }^{12}$ Appliances are intended either for continuous wear (except for meals and oral hygiene), night-only wear, or to be used several times a day for a short period of time (10-20 minutes) in conjunction with exercise and/or massage. ${ }^{4}$

Some disadvantages of these appliances include pressure sores, the potential for skin breakdown at the commissures especially with the use of horizontal stretching appliances, appliance breakage from prolonged stress, drooling, and patient compliance. ${ }^{16,17}$

\section{CASE REPORT}

A 16-year-old male patient presented with a complaint of limited mouth opening after a facial burn involving the perioral soft tissues approximately 2 months earlier (Figure 1). Until that point, a microstomia prevention regimen had been put in place, which included daily exercises and massage and the patient monitored and assessed to determine whether appliance usage would be indicated if there were early signs of contracture development. At this point, based on the complaint of the patient, the addition of an appliance was deemed appropriate to complement the existing treatment. Given that a few weeks had already passed after the initial injury, it was considered paramount to start with appliance treatment without any additional delay.

The planning and design of the microstomia prevention appliance and the wear regimen was performed by a multidisciplinary team. This included a plastic surgeon, orthodontist/dentist, dental technician, physiotherapist, and occupational therapist. A dynamic appliance was desired that would provide both horizontal and vertical stretching with adjustable forces.

A dynamic appliance was fabricated using acrylic resin, expansion screws, and springs, providing simultaneous horizontal and vertical circumoral forces. Appliance construction was easy and inexpensive without the need for taking impressions, which also ensured no delay for delivery and little oral manipulation required for fabrication. Adjustment was possible so that it was almost painlessly inserted and subsequently progressively activated. A dental technician was available on site and the presence of an in-house dental laboratory facilitated appliance construction. Since impression taking is not necessary however one could imagine prefabricating these appliances and making any necessary adjustments in the clinic. 

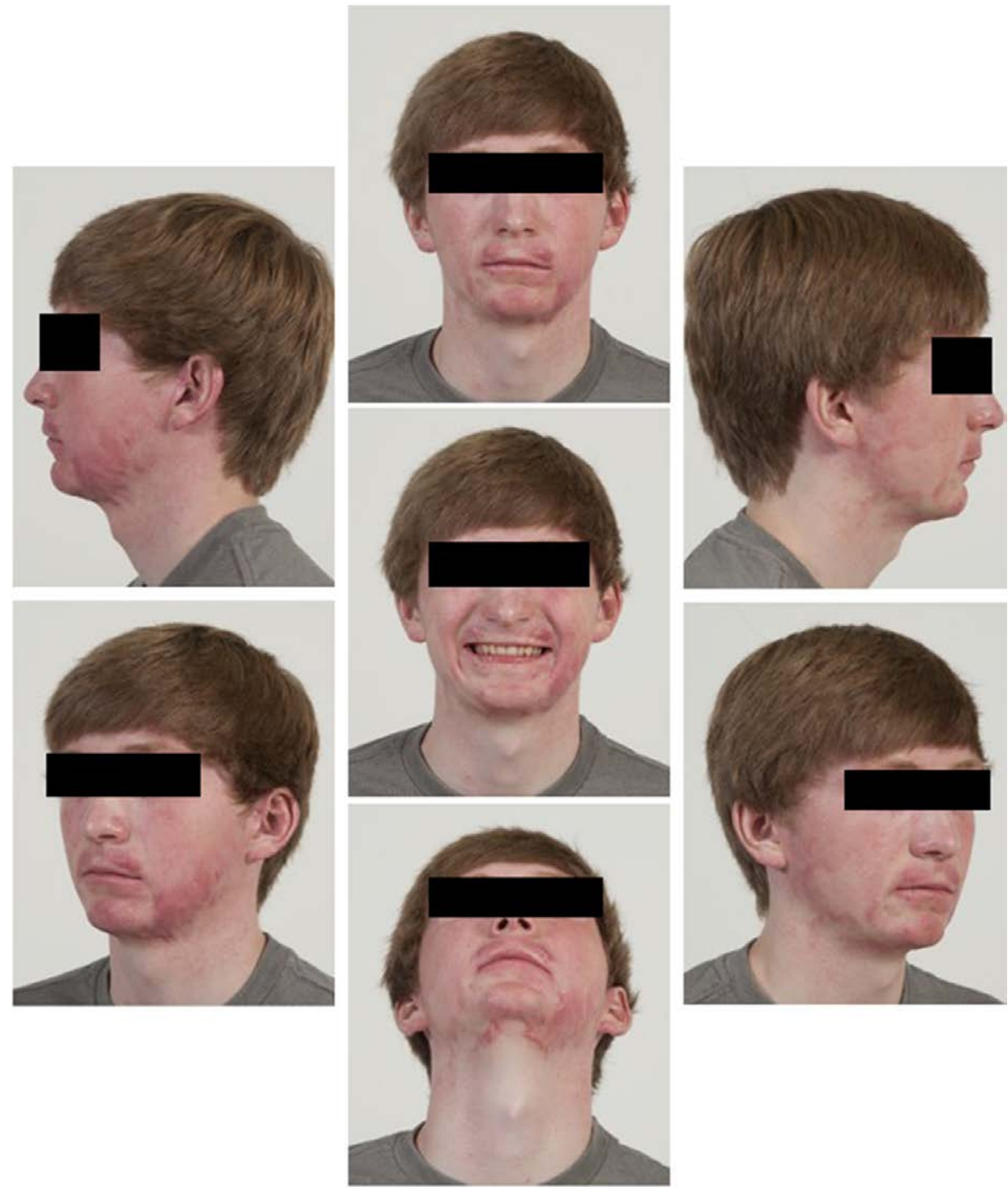

Figure 1. Extraoral facial photographs before appliance insertion showing extent of burn injury.

The design of the appliance in situ (Figure 2) is such that there are four acrylic pads that are in contact with the two oral commissures and the center of the upper and lower lips. Expansion screws with springs are inserted so that the appliance is adjustable both horizontally and vertically up until the desired extent. It is convenient for use because the patient controls the pressure that is applied by the appliance.
The patient was instructed to massage the commissural tissues approximately 5 minutes before appliance insertion. The appliance was inserted in a passive deactivated state, and this was followed by a slow-controlled opening (activation) up to a point the patient could tolerate without excessive discomfort. The wearing regimen consisted of appliance use for 15 to 30 minutes for 2 to 4 sessions per day,
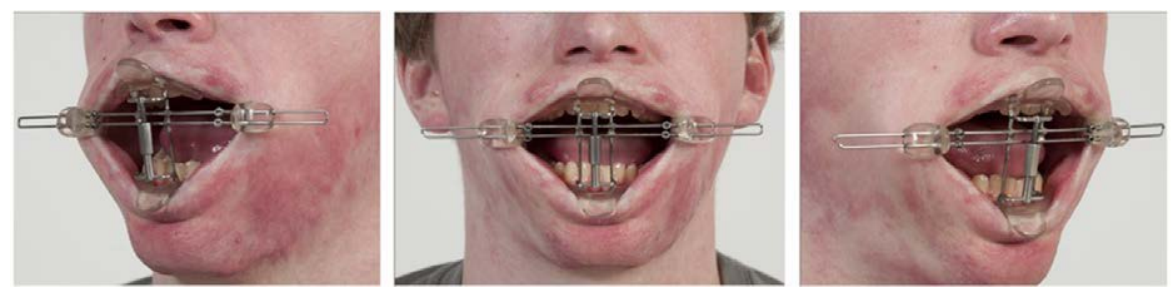

Figure 2. Extraoral perioral photographs showing appliance in place on the day of insertion. 
depending on the patient's daily tolerance. For appliance removal, a slow-controlled closing (deactivation) of the appliance was possible. The wear schedule was decreased based on how well the patient could maintain the improved opening. The patient was asked to concentrate initially, for the first couple of months, on horizontal stretching so as to stretch the commissures. Vertical stretching was progressively added afterward. Once range of motion was stabilized, the appliance was discontinued and the patient continued to perform facial exercises and scar massage. The total duration of appliance wear was approximately 10 months.

Follow-up appointments were quick with no appliance adjustments necessary. Both the horizontal and the vertical distance could be measured at baseline and at each follow-up appointment to record the progress. This was simply performed by measuring the distance between the acrylic pads at maximum activation. Baseline and progress recordings of horizontal and vertical openings showed slow and steady progression with a total increase of about $26 \mathrm{~mm}$ horizontal and $17 \mathrm{~mm}$ vertical opening compared with baseline (Figure 3).

Throughout the period of use of the appliance, the patient reported comfort, ease of use, and overall satisfaction. Functional outcomes, although not directly measured, were also improved based on reports of the patient and his parents.

\section{DISCUSSION}

Management of microstomia is a critical area when treating a patient with burn injuries and should be a priority due to its impact on quality of life. A plethora of different microstomia prevention appliances have been presented in the literature during the past decades. ${ }^{4}$ Arriving at a reasonable decision as to which appliance should be used in the clinical management of these patients should be planned jointly by the physicians, orthodontists/dentists, therapists, and any other health professionals who are familiar with the significant variables affecting the efficacy and effectiveness of the appliances. ${ }^{18}$ In the present case, the presence of the dental technician in the decision-making process was vital as the conception, design, and fabrication of the appliance were performed by the in-house dental technician and without having to recall the patient for an appliance insertion appointment. The present appliance was used in a mild microstomia case but

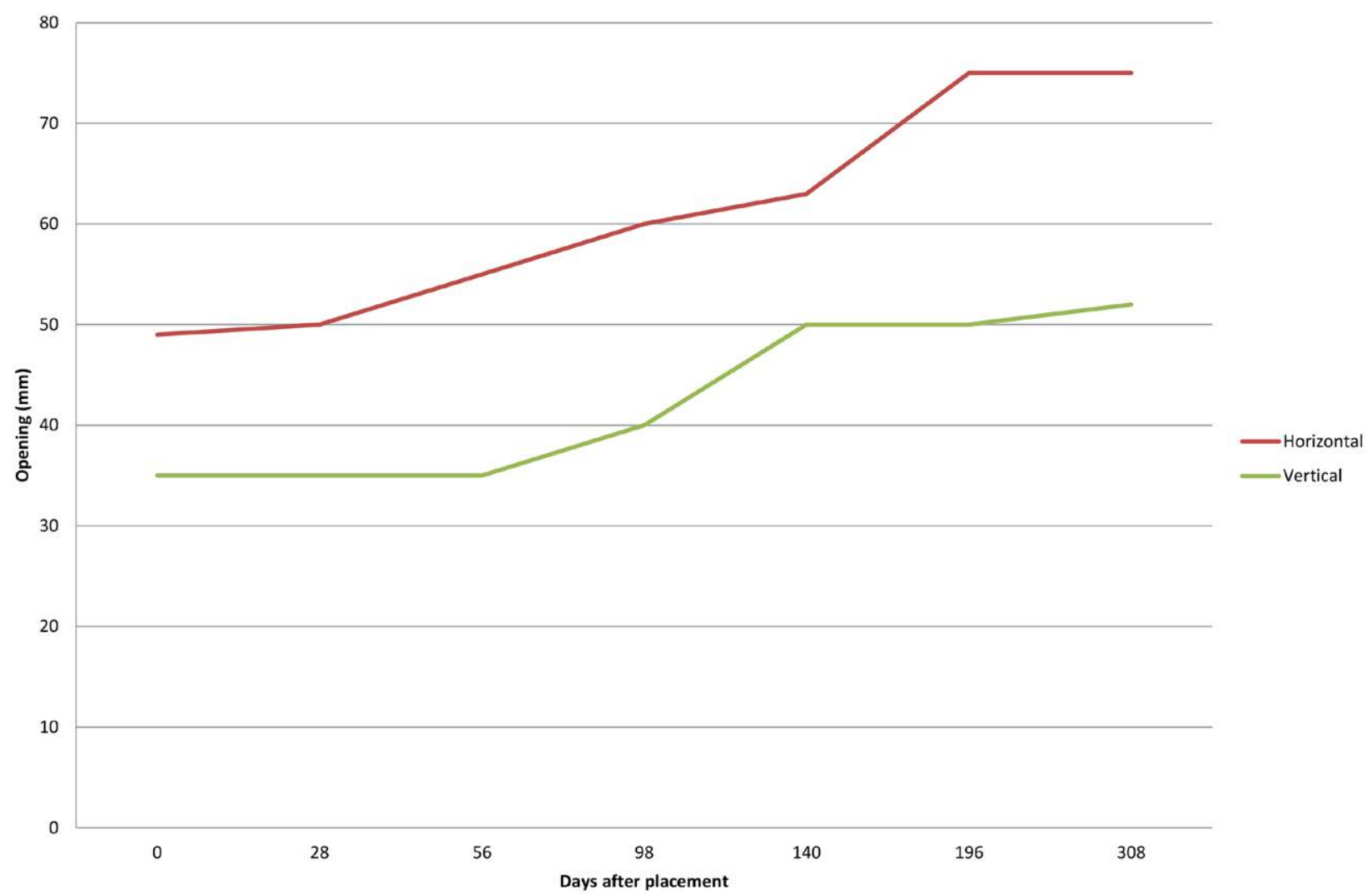

Figure 3. Line graph showing recordings of horizontal and vertical openings and their evolution over time throughout the period of appliance wear, from the day of placement to approximately 10 months after placement. 
could have the potential to be used also in moderate to severe microstomia cases, although this has yet to be tried.

Reduced vertical mouth openings present a limit to the use of many appliances, as one needs to be able to get the appliance in even with very limited mouth opening at first. ${ }^{15}$ The activation screw on the vertical component of our appliance is a big advantage as patients can insert the appliance fully deactivated and then perform a slowcontrolled opening of the expansion screw, which comfortably stretches the tissues. This also has the potential advantage of reducing pain since the appliance can be inserted in a passive manner and subsequently activated.

Other advantages of the present appliance include the even distribution of pressure and tissue stretching due to the presence of four acrylic pads, which help decrease the potential of developing pressure sores. The vertical forces acting on soft tissues, as opposed to the teeth, ensure that risk of side effects from applying excessive forces to the upper and lower incisors is eliminated. In addition, the vertical forces of the appliance provide the stretch on the soft tissues responsible for microstomia. A small number of follow-up appointment sessions are required and the duration of each appointment is also short because no appliance adjustment is necessary. This is beneficial to the patient and the family. Comfort is also assured, which can improve patient compliance by promoting easier adherence to the appliance wear regimen.

When the appliance is used as recommended, good results in functional mouth opening can be obtained. This improvement can have an impact on the patients' psychosocial well-being and quality of life by enabling them to perform activities such as speech, eating, dental hygiene, facial expressions, and social interactions.

Functional outcomes should be measured and recorded to keep track of improvements. Ideally, preburn commissural dimensions must be determined to achieve the most aesthetic and functional results after healing, ${ }^{12}$ but these dimensions are rarely available to the treating healthcare team. Instead of relying on these unobtainable measurements, one can rely on measuring dimensions at the time of appliance insertion or treatment introduction to be able to appraise improvements observed. One of the first methods proposed to assess microstomia measured the distance from the incisal edge of the right upper central incisor to the incisal edge of the right lower central incisor. ${ }^{19}$ However, it is also necessary to assess soft tissue aperture, measuring the largest vertical distance between the upper and lower lip and the distance between the two commissures of the mouth. The magnitude of oral aperture is a determining factor for diagnosis and may have implications for the treatment of these patients.

Given the myriad of microstomia prevention appliances that have been described in the literature, selection of an appropriate appliance is challenging. The appliance presently described offers several advantages including the application of both vertical and horizontal forces, ease of construction and adjustments, and comfort for the patient. Since compliance is an essential component of treatment success, an appliance well tolerated by the patient may often be the most appropriate.

\section{CONCLUSIONS}

The use and construction of a postburn microstomia prevention appliance is presented and has improved mouth opening and consequently functional outcomes in a 16-year-old boy, while upholding patient satisfaction throughout treatment.

\section{REFERENCES}

1. Heinle JA, Kealey GP, Cram AE, Hartford CE. The microstomia prevention appliance: 14 years of clinical experience. J Burn Care Rehabil 1988;9:90-1.

2. Morgan RF, Nichter LS, Haines PC, Kenney JG, Friedman HI, Edlich RF. Management of head and neck burns. J Burn Care Rehabil 1985;6:20-38.

3. McGowan RH. Prevention of microstomia following facial burns. Br Dent J 1980;149:83-4.

4. Dougherty ME, Warden GD. A thirty-year review of oral appliances used to manage microstomia, 1972 to 2002. J Burn Care Rehabil 2003;24:418-31.

5. Garnett MJ, Nohl FS, Barclay SC. Management of patients with reduced oral aperture and mandibular hypomobility (trismus) and implications for operative dentistry. Br Dent J 2008;204:125-31.

6. Nussbaum BL. Dental care for patients who are unable to open their mouths. Dent Clin North Am 2009;53:323-8, x.

7. Silverglade D, Ruberg RL. Nonsurgical management of burns to the lips and commissures. Clin Plast Surg 1986;13:87-94.

8. Larson DL, Abston S, Evans EB, Dobrkovsky M, Linares HA. Techniques for decreasing scar formation and contractures in the burned patient. J Trauma 1971;11:807-23.

9. Pizzo G, Scardina GA, Messina P. Effects of a nonsurgical exercise program on the decreased mouth opening in patients with systemic scleroderma. Clin Oral Investig 2003;7:175-8.

10. Zweifel CJ, Guggenheim M, Jandali AR, Altintas MA, Künzi W, Giovanoli P. Management of microstomia in adult burn patients revisited. J Plast Reconstr Aesthet Surg 2010;63:e351-7

11. Makiguchi T, Yokoo S, Koitabashi A, Ogawa M, Miyazaki $\mathrm{H}$, Terashi $\mathrm{H}$. Treatment of microstomia caused by burn with a nasolabial flap - an ingenious approach for tugging 
and fixation of the oral commissure. J Craniofac Surg 2014;25:568-70.

12. Taylor LB, Walker J. A review of selected microstomia prevention appliances. Pediatr Dent 1997;19:413-8.

13. Silvestre-Rangil J, Martinez-Herrera M, Silvestre FJ. Dental management of patients with microstomia. A review of the literature and update on the treatment. J Oral Res 2015;4:340-50.

14. Gay WD. Prostheses for oral burn patients. J Prosthet Dent 1984;52:564-6.

15. Wust KJ. A modified dynamic mouth splint for burn patients. J Burn Care Res 2006;27:86-92.
16. Carlow DL, Conine TA, Stevenson-Moore P. Static orthoses for the management of microstomia. J Rehabil Res Dev 1987;24:35-42.

17. Penna KJ, Sadoff RS. Prevention of microstomia following facial burns. Br J Oral Maxillofac Surg 1998;36:146-7.

18. Conine TA, Carlow DL, Stevenson-Moore P. Dynamic orthoses for the management of microstomia. J Rehabil Res Dev 1987;24:43-8.

19. Naylor WP, Douglass CW, Mix E. The nonsurgical treat ment of microstomia in scleroderma: a pilot study. Oral Surg Oral Med Oral Pathol 1984;57:508-11. 